\title{
Congress
}

\section{Unusual patterns of oestrus}

\section{Angelika Von Heimendahl}

Oestrus patterns and the length of oestrus can vary between bitches and also within the lifetime of a female. This can be of great concern to breeders, who see any irregularity as a sign of possible infertility. The lecture will discuss:

- Persistent oestrus in young animals

- Persistent oestrus in older animals

- Oestrus after ovariohysterectomy

- Delayed puberty or no oestrus observed in a young healthy female

- Interoestrus intervals which are shorter than usual (less than 3 months)

- Interoestrus intervals which are longer than usual (more than 12 months).

The canine oestrous cycle is unusual within the mammal world. Long periods of anoestrus are followed by many days of pro-oestrus and oestrus. When ovulation occurs the oocytes are not ready to be fertilized and remain in the oviduct for a prolonged period of time, unknown in other mammals.

Persistent oestrus in young animals is very different from the same pattern in older animals. There are always many questions about the age a bitch should have gone through puberty and what the limits of a normal fertile interoestrus interval is. Reassuring breeders that some of the more unusual patterns are still within normal reproductive parameters and have no impact on future fertility can go a long way to establishing a good relationship.

A sound knowledge of the wide normal and abnormal patterns within the canine oestrous cycle is necessary to advise breeders when to mate, when to wait and when it is unlikely that this cycle will produce viable oocytes.

The lecture will explain different methods to monitor unusual patterns of oestrus as well as induction of and delaying oestrus pharmacologically.

\section{KEY LEARNING OBJECTIVES}

- Limits of normal oestrus parameters in the canine

- Monitoring unusual oestrus patterns without spending too much money

- Ovarian remnant syndrome

\section{MULTIPLE CHOICE QUESTIONS}

1. A 7-month-old Cocker Spaniel bitch is presented having shown swelling of the vulva and haemorrhagic vaginal discharge for 5 weeks. The owners would like to breed from her in the future. What advice should be given?

(A) The bitch shows an unusual pattern of oestrus at a young age and is not suitable for breeding

(B) The bitch is showing signs of an unovulatory season which is normal in puberty and will be unlikely to have any problems in the future

(C) The bitch should be treated with long-acting progesterone to stop the bleeding and her future breeding potential is questionable

(D) The bitch should have an ovariohysterectomy as she is at the right age and should not be bred from

2. An 8-year-old bitch, spayed 4 years ago, attracts the interest of males every 7 months. Her vulva is slightly swollen, but there is no vaginal discharge. What is a likely cause of this?

(A) Lack of oestrogen causes vaginitis and this may be attractive to the males

(B) Behavioural problem as an ovarian remant would have been obvious much sooner after the spay

(C) Other endocrinological active tissues may be producing oestrogen

(D) An ovarian remnant is the most likely cause and vaginal cytology will confirm this

3. A German Shepherd bitch has interoestrus intervals of less than 4 months. The breeder has tried to breed from her but she has not become pregnant. What advice should be given about the impact the short interoestrus intervals can have on fertility?

(A) Short interoestrus intervals are not uncommon in German Shepherd dogs and have no impact on fertility

(B) Short interoestrus intervals can have a negative impact on fertility because the endometrium has no time to recover

(C) Short interoestrus intervals give the breeder more opportunities to mate the bitch

(D) Short interoestrus intervals mean that the bitch cannot get pregnant 